\title{
Canonical Strangeness and Distillation Effects in Hadron Production
}

\author{
V.D. Toneev ${ }^{1,2}$ and A.S. Parvan ${ }^{2,3}$ \\ 1 Gesellschaft für Schwerionenforschung mbH, 64291 Darmstadt, Germany \\ 2Joint Institute for Nuclear Research, 141980 Dubna, Russia and \\ 3Institute of Applied Physics, Moldova Academy of Sciences, MD-2028 Kishineu, Moldova \\ Strangeness canonical ensemble for Maxwell-Boltzmann statistics is reconsidered \\ for excited nuclear systems with non-vanishing net strangeness. A new recurrence \\ relation method is applied to find the partition function. The method is first gen- \\ eralized to the case of quantum strangeness canonical ensemble. Uncertainties in \\ calculation of the $K^{+} / \pi^{+}$excitation function are discussed. A new scenario based \\ on the strangeness distillation effect is put forward for a possible explanation of \\ anomalous strangeness production observed at the bombarding energy $\sim 30 \mathrm{GeV}$. \\ The peaked maximum in the $K^{+} / \pi^{+}$ratio is considered as a sign of the critical end- \\ point reached in evolution of the system rather than a latent heat jump emerging \\ from the onset of the first order deconfinement phase transition.
}

\section{INTRODUCTION}

In recent years, statistical thermal models have widely been used for analyzing heavy ion collisions (see the review article [1]). These models describe available phase space at the final stage of nuclear collision when inelastic interactions between hadrons cease, i.e., at the chemical freeze-out. The grand canonical (GC) ensemble is defined in the large volume limit, so the volume parameter $V$ is an extensive coefficient in the expressions for such quantities as mean particle numbers controlled by the chemical potentials. Application of the GC ensemble also implies that the number of produced particles carrying a conserved charge is sufficiently large. In this description the net value of a given charge (e.g. electric charge, baryon number, strangeness, charm etc.) fluctuates from event to event. These multiplicity fluctuations with respect to mean particle multiplicity can be neglected only if the particles carrying the charges are abundant, i.e., for the system of a large volume 
and/or high temperature. Here the charge is conserved on the average and, therefore, the GC description is adequate.

In the opposite limit of low production rate, fluctuations of the particle number can be as large as its event averaged value. In this case, the charge conservation has to be implemented exactly in each event. So the ensemble to be chosen is the canonical one. Then, the $V$ independent fugacities are replaced by nonlinearly $V$-dependent canonical chemical factors. The exact conservation of quantum numbers introduces certain constraints on the system considered. In the formulation of thermodynamics of strongly interacting matter one needs to implement these constraints that are originally governed by internal symmetry of the Hamiltonian [2, 3, 4]. Consequently, the equilibrium distribution of particle multiplicities can differ from that expected in the GC limit.

In principle, for an equilibrium system all conserved charges should be treated canonically. However, in a good approximation, baryonic and electric charge conservation can be considered grand-canonically even for high-energy $p p$ collisions. This treatment results only in a slight overestimation of the canonical effects [5]. On the other hand, the importance of exact strangeness conservation for hadron production in heavy ion collisions has been clearly demonstrated some time ago in Refs. [6, 7], where closed expressions for suppression of chemical factors were derived in terms of classical Maxwell-Boltzmann statistics for the global strangeness neutrality condition $S=0$.

In this paper, we reconsider the derivation of the strangeness constraint for nuclear systems with non-vanishing net strangeness $S$ applying two different methods for evaluating the partition function and ensemble averages: the direct method and the method of recurrence relations [8]. In the line of Refs. [9, 10], the second method allows us first to generalize our canonical strangeness evaluation to the case of quantum statistics. Two Appendixes contain details on the calculation of canonical quantities by recurrence relations. The derived formulae will be used to analyze the kaon excitation function from heavy ion collisions and particular attention will be paid to the discussion of an anomalous maximum of excitation functions at the beam energy near $30 \mathrm{GeV}$. A new possible scenario of its formation as a manifestation of the critical end-point is advanced. 


\section{CLASSICAL STATISTICS}

Let us construct the thermodynamic partition function $Z_{S}$ with the exact strangeness conservation for a relativistic perfect gas of $N$ hadron species at temperature $T$ in space volume $V$. Every hadron species $\alpha(\alpha=1, \ldots, N)$ is characterized by the baryonic $b_{\alpha}$, electric $q_{\alpha}$ and strangeness $s_{\alpha}$ charges, the mass $m_{\alpha}$ and the spin degeneration factor $g_{\alpha}$. Let us assume that this particle ensemble is described by the Maxwell-Boltzmann statistics. In the occupation number $\left\{\nu_{\alpha p}\right\}$ representation, the constraint for an exact conservation of the net strangeness $S$ can be directly imposed on the grand-canonical partition function conserving the average baryonic and electric charges :

$$
Z_{S}=\sum_{\left\{\nu_{\alpha p}\right\}} \delta\left(\sum_{\alpha, p} s_{\alpha} \nu_{\alpha p}-S\right) \frac{1}{\prod_{\alpha, p} \nu_{\alpha p} !} e^{-\beta \sum_{\alpha, p} \nu_{\alpha p} \varepsilon_{\alpha p}}
$$

with

$$
\varepsilon_{\alpha p}=\sqrt{\vec{p}^{2}+m_{\alpha}^{2}}-\mu_{B} b_{\alpha}-\mu_{Q} q_{\alpha} .
$$

Here $\beta=1 / T, \mu_{B}, \mu_{Q}$ are baryonic and electric chemical potentials, respectively, and multiindex $p \equiv(\vec{p}, \sigma, \ldots)$ includes particle momentum $\vec{p}$, spin projection $\sigma$ and other quantum numbers. The summation in (11) is carrying out over all permutations $\left\{\nu_{\alpha p}\right\}$ of occupation numbers. In this representation, the ensemble average for the operator $O\left(\left\{\nu_{\alpha p}\right\}\right)$ is defined as follows :

$$
\langle O\rangle_{S}=\frac{1}{Z_{S}} \sum_{\left\{\nu_{\alpha p}\right\}} \delta\left(\sum_{\alpha, p} s_{\alpha} \nu_{\alpha p}-S\right) O\left(\left\{\nu_{\alpha p}\right\}\right) \frac{1}{\prod_{\alpha, p} \nu_{\alpha p} !} e^{-\beta \sum_{\alpha, p} \nu_{\alpha p} \varepsilon_{\alpha p}} .
$$

These basic expressions will be used below for finding the mean occupation numbers and mean particle multiplicities.

\section{A. The direct method for solving the partition function}

The partition function $Z_{S}$ can be summed up over the momentum $\vec{p}$ by inserting the identical unit in the form $\sum_{\left\{n_{\alpha}\right\}} \prod_{\alpha} \delta\left(\sum_{p} \nu_{\alpha p}-n_{\alpha}\right)=1$ into Eq.(1). Then, the partition function is reduced to

$$
Z_{S}=\sum_{\left\{n_{\alpha}\right\}} \delta\left(\sum_{\alpha} s_{\alpha} n_{\alpha}-S\right) \prod_{\alpha} \frac{\left(Z_{\alpha}^{1}\right)^{n_{\alpha}}}{n_{\alpha} !}
$$


where the grand-canonical single particle partition function

$$
Z_{\alpha}^{1}=\sum_{p} e^{-\beta \varepsilon_{\alpha p}}=e^{\beta\left(\mu_{B} b_{\alpha}+\mu_{Q} q_{\alpha}\right)} \frac{g_{\alpha} V}{(2 \pi)^{3}} \int d^{3} \vec{p} e^{-\beta \sqrt{\vec{p}^{2}+m_{\alpha}^{2}}} .
$$

Using the integral representation for the Kronecker symbol in Eq.(41), one can carry out summation over all particle numbers $\left\{n_{\alpha}\right\}$ by means of series expansion of the exponent. So we have

$$
Z_{S}=\frac{1}{2 \pi} \int_{0}^{2 \pi} d \phi \exp \left[-\imath S \phi+\sum_{\alpha} Z_{\alpha}^{1} e^{\imath s_{\alpha} \phi}\right] .
$$

One should note that this representation of $Z_{S}$ based on the classical statistical partition function (11) coincides exactly with the starting expression used in [1, 6, 7].

Let us proceed now from the summation over species index $\alpha$ to the sum over all available strangeness values $s$ for individual particles in the system. So we substitute into Eq. (6) the identical unit in the form $\sum_{s=-s_{\max }}^{s_{\max }} \delta_{s, s_{\alpha}}=1$, which is valid for any species $\alpha$ under condition $\left|s_{\alpha}\right| \leq s_{\max }$

$$
Z_{S}=\frac{1}{2 \pi} \int_{0}^{2 \pi} d \phi \exp \left[-\imath S \phi+\sum_{s=-s_{\max }}^{s_{\max }} S_{s} e^{\imath s \phi}\right]
$$

where

$$
S_{s}=\sum_{\alpha} \delta_{s, s_{\alpha}} Z_{\alpha}^{1}, \quad-s_{\max } \leq s \leq s_{\max }
$$

By rewriting the exponent of the sum over $-s_{\max } \leq s \leq s_{\max }$ in Eq.(7) as the product of exponents and expanding every exponent into series, $e^{x}=\sum x^{n} / n$ !, we get

$$
\exp \left[\sum_{s} S_{s} e^{\imath s \phi}\right]=\sum_{\left\{N_{s}\right\}} \exp \left[\sum_{s} \imath s N_{s} \phi\right] \prod_{s} \frac{S_{s}^{N_{s}}}{N_{s} !},
$$

where $N_{s}$ are non-negative integer numbers. Here the following short-hand notation has been used for the sum $\sum_{\left\{N_{s}\right\}} \equiv \sum_{N_{-s_{\max }}} \ldots \sum_{N_{s_{\max }}}$. After substitution of Eq.(9]) into (77) and integration with respect to $\phi$, the strangeness canonical partition function $Z_{S}$ is reduced to the following equation :

$$
Z_{S}=\sum_{\left\{N_{s}\right\}} \delta\left(\sum_{s=-s_{\max }}^{s_{\max }} s N_{s}-S\right) \prod_{s=-s_{\max }}^{s_{\max }} \frac{S_{s}^{N_{s}}}{N_{s} !}
$$

If the integral representation for the Kronecker symbol is used in Eq.(10), we come back to Eq.(7) for the partition function in the statistical model [1]. 
A number of sums over the set $\left\{N_{s}\right\}$ in Eq.(10) can be reduced. For this one should introduce the identity

$$
\prod_{s=1}^{s_{\max }} \sum_{n_{s}=-\infty}^{\infty} \delta\left(N_{s}-N_{-s}-n_{s}\right)=1
$$

into (10), to be valid for every fixed number set $\left\{N_{s}\right\}$, and change the order of summing with respect to $\left\{n_{s}\right\}$ and $\left\{N_{s}\right\}$. Then, using the Kronecker symbol in Eq.(111), the summation for the set $\left\{N_{s}\right\}$ can be done explicitly. It is noticed that these sums are grouped by pairs for every $\mp|s|$ and every pair results in the modified Bessel functions. Finally, for Eq.(10) we get

$$
Z_{S}=e^{S_{0}} \sum_{n_{1}=-\infty}^{\infty} \ldots \sum_{n_{s_{\max }}=-\infty}^{\infty} \delta\left(\sum_{s=1}^{s_{\max }} s n_{s}-S\right) \prod_{s=1}^{s_{\max }} a_{s}^{n_{s}} I_{-n_{s}}\left(x_{s}\right),
$$

where $I_{n}$ is the modified Bessel function and variables

$$
x_{s}=2 \sqrt{S_{-s} S_{s}}, \quad a_{s}=\sqrt{S_{s} / S_{-s}}, \quad s=1, \ldots, s_{\max } .
$$

Taking into account Eq.(8) we see that $x_{s} \sim V$ while $a_{s}$ are independent of the system volume. In specific calculations the maximal hadron strangeness $s_{\max }$ is not large, which simplifies essentially Eq.(12), since the number of infinite sums is $\left(s_{\max }-1\right)$. In particular, for $s_{\max }=1,2,3$ the partition function (12) can be expressed as follows :

$$
\begin{aligned}
& Z_{S}=e^{S_{0}} \sum_{n=-\infty}^{\infty} \sum_{m=-\infty}^{\infty} a_{1}^{S-2 n-3 m} a_{2}^{n} a_{3}^{m} I_{S-2 n-3 m}\left(x_{1}\right) I_{n}\left(x_{2}\right) I_{m}\left(x_{3}\right), \quad s_{\max }=3 \\
& Z_{S}=e^{S_{0}} \sum_{n=-\infty}^{\infty} a_{1}^{S-2 n} a_{2}^{n} I_{S-2 n}\left(x_{1}\right) I_{n}\left(x_{2}\right), \quad s_{\max }=2 \\
& Z_{S}=e^{S_{0}} a_{1}^{S} I_{S}\left(x_{1}\right), \quad s_{\max }=1
\end{aligned}
$$

where the symmetry property of the modified Bessel function $I_{-n}=I_{n}$ has been used. Associative production of multi-strange baryons is neglected in Eq.(16). This simplified equation for $S \neq 0$ in the case of $s_{\max }=1$ has been derived earlier in [1]. The result for $s_{\max }=4$ can be found in [11]. The case of full canonical treatment of both baryon and strangeness $S \neq 0$ in a little bit different technique was given in [12]. However, the electric charge conservation is taken into account by neither canonically, nor grand canonically. It simplifies calculations but the final result differs from our Eqs.(14)-(16). The single particle partition function $Z_{\alpha}^{1}$ given by Eq.(5) can be integrated resulting in the well-known expression

$$
Z_{\alpha}^{1}=\frac{g_{\alpha} V}{2 \pi^{2}} \frac{m_{\alpha}^{2}}{\beta} K_{2}\left(\beta m_{\alpha}\right) e^{\beta\left(\mu_{B} b_{\alpha}+\mu_{Q} q_{\alpha}\right)}
$$

with the Bessel function $K_{2}$. 


\section{B. Method of recurrence relations}

Let us subdivide the set $M$ of particles of all species into three sub-sets $M_{ \pm}$and $M_{0}$ according to the value of individual strangeness charge $s_{\alpha}$ of involved hadrons. For a fixed set of occupation numbers $\left\{\nu_{\alpha p}\right\}$ we introduce notation of positive $S_{+}$and negative $S_{-}$ components of the net strangeness $S$ of the system

$$
\sum_{\alpha \in M_{\mp}} \sum_{p}\left|s_{\alpha}\right| \nu_{\alpha p}=S_{\mp}
$$

where $M_{+}$and $M_{-}$are the sets of all species $\alpha$ for which $s_{\alpha}>0$ and $s_{\alpha}<0$, respectively. For the zero component the following equality is fulfilled $\sum_{\alpha \in M_{0}} \sum_{p}\left|s_{\alpha}\right| \nu_{\alpha p}=0$, since $M_{0}$ is the set of all non-strange hadrons, $s_{\alpha}=0$. Let us substitute into Eq.(11) the product of two identical unit operators corresponding to a fixed set of occupation numbers $\left\{\nu_{\alpha p}\right\}$ :

$$
\sum_{S_{\mp}=0}^{\infty} \delta\left(\sum_{\alpha \in M_{\mp}} \sum_{p}\left|s_{\alpha}\right| \nu_{\alpha p}-S_{\mp}\right)=1
$$

Then for the strangeness canonical partition function we have

$$
Z_{S}=Z^{(0)} \sum_{S_{+}=0}^{\infty} \sum_{S_{-}=0}^{\infty} \delta\left(-S_{-}+S_{+}-S\right) Z_{S_{+}}^{(+)} Z_{S_{-}}^{(-)},
$$

where the partition functions for positive $Z_{S_{+}}^{(+)}$and negative $Z_{S_{-}}^{(-)}$components of the net strangeness $S$ have the following form in the case of classical statistics :

$$
Z_{S_{\mp}}^{(\mp)}=\sum_{\left\{\nu_{\alpha p}\right\}_{\alpha \in M_{\mp}}} \delta\left(\sum_{\alpha \in M_{\mp}} \sum_{p}\left|s_{\alpha}\right| \nu_{\alpha p}-S_{\mp}\right) \frac{1}{\prod_{\alpha \in M_{\mp}} \prod_{p} \nu_{\alpha p} !} e^{-\beta \sum_{\alpha \in M_{\mp}} \sum_{p} \nu_{\alpha p} \varepsilon_{\alpha p}} .
$$

The positive $S_{+}$and negative $S_{-}$strangeness components take integer non-negative values. The appropriating partition functions can be represented as follows (see Appendix A) :

$$
Z_{S_{\mp}}^{(\mp)}=\frac{1}{S_{\mp}} \sum_{\alpha \in M_{\mp}}\left|s_{\alpha}\right| Z_{\alpha}^{1} Z_{S_{\mp}-\left|s_{\alpha}\right|}^{(\mp)}=\frac{1}{S_{\mp}} \sum_{s=1}^{s_{\max }} s S_{\mp s} Z_{S_{\mp}-s}^{(\mp)}
$$

where the second equality in (22) was obtained by introducing once more identical unit in the form $\sum_{s=1}^{s_{\max }} \delta_{s,\left|s_{\alpha}\right|}=1$, to be valid for any species $\alpha$ under condition $\left|s_{\alpha}\right| \leq s_{\max }$. Here by definition $Z_{0}^{(\mp)}=1, s_{\max }=\max \left\{\left|s_{\alpha}\right|\right\}$ is maximal modulus value of particle strangeness in the given system and auxiliary functions are defined as

$$
S_{\mp s}=\sum_{\alpha \in M_{\mp}} \delta_{s,\left|s_{\alpha}\right|} Z_{\alpha}^{1}, \quad 1 \leq s \leq s_{\max } .
$$


It is of great interest that mathematical structure of Eqs.(22) allows one an exact recursive solution because the partition function of the given strangeness component $Z_{S_{\mp}}^{(\mp)}$ is expressed through the sum of partition functions but with smaller index of the strangeness components. This equation can be solved iteratively starting from the lowest component $Z_{0}^{(\mp)}=1$. For calculating $Z_{S_{\mp}}^{(\mp)}$ this procedure converges quickly as is seen from Eq.(21): High values of $S_{\mp}$ in the argument of the delta function should be compensated by large occupation numbers which are exponentially suppressed with increasing negative exponent. This situation reminds very much of finding the exactly solvable partition function in the nuclear multifragmentation model [9, 10, 13] and in the context of quark-gluon plasma [14] where the method of recurrence relations turned out to be very powerful.

The partition function $Z^{(0)}$ with zero component of net strangeness $S$ is reduced to the following generating function $Z^{(0)}=e^{S_{0}}$ with $S_{0}=\sum_{\alpha \in M_{0}} Z_{\alpha}^{1}$, according to Eq.(23).

\section{Ensemble averages}

The mean occupation numbers can be found as ensemble averages (3) for the operator $O\left(\left\{\nu_{\alpha p}\right\}\right)=\nu_{\alpha p}$. Using mathematical technique for summing over occupation numbers as developed in [9, 10], we get for the mean occupation numbers the following result (see Appendix A) :

$$
\left\langle\nu_{\alpha p}\right\rangle_{S}=e^{-\beta \varepsilon_{\alpha p}} \frac{Z_{S-s_{\alpha}}}{Z_{S}} .
$$

The mean particle number of species $\alpha$ is obtained by summing the mean occupation numbers (24) over momentum $\vec{p}$

$$
\left\langle n_{\alpha}\right\rangle_{S}=\sum_{p}\left\langle\nu_{\alpha p}\right\rangle_{S}=Z_{\alpha}^{1} \frac{Z_{S-s_{\alpha}}}{Z_{S}} .
$$

Eq.(25) was given also in [11]. It is easy to convince ourselves that the mean number of particles with strangeness $s$ in the system with the net strangeness $S$ can be represented as follow :

$$
\left\langle N_{s}\right\rangle_{S}=\sum_{\alpha} \delta_{s, s_{\alpha}}\left\langle n_{\alpha}\right\rangle_{S}=S_{s} \frac{Z_{S-s}}{Z_{S}}
$$

with $S_{s}$ defined by Eq.(23). As a particular case, for the particle density in strangeness canonical ensemble $n_{\alpha}=\left\langle n_{\alpha}\right\rangle_{S} / V$ we have: 


$$
\begin{aligned}
& n_{\alpha}=\frac{Z_{\alpha}^{1}}{V} \frac{e^{S_{0}}}{Z_{S}} \sum_{n=-\infty}^{\infty} \sum_{m=-\infty}^{\infty} a_{1}^{S-s_{\alpha}-2 n-3 m} a_{2}^{n} a_{3}^{m} I_{S-s_{\alpha}-2 n-3 m}\left(x_{1}\right) I_{n}\left(x_{2}\right) I_{m}\left(x_{3}\right), s_{\max }=3,(2), \quad s_{\max }=2, \\
& n_{\alpha}=\frac{Z_{\alpha}^{1}}{V} \frac{e^{S_{0}}}{Z_{S}} \sum_{n=-\infty}^{\infty} a_{1}^{S-s_{\alpha}-2 n} a_{2}^{n} I_{S-s_{\alpha}-2 n}\left(x_{1}\right) I_{n}\left(x_{2}\right), \quad s_{\max }=1 . \\
& n_{\alpha}=\frac{Z_{\alpha}^{1}}{V}\left(\frac{S_{-1}}{\sqrt{S_{-1} S_{1}}}\right)^{s_{\alpha}} \frac{I_{S-s_{\alpha}}\left(x_{1}\right)}{I_{S}\left(x_{1}\right)}, \quad
\end{aligned}
$$

Similarly to Eqs.(14)-(16) only Eq.(27) takes into account that strangeness of, for example, kaons with $s_{\alpha}=1$ can be compensated associatively by production of any multi-strange hyperon. For non-strange hadrons, $s_{\alpha}=0$, the particle density is

$$
n_{\alpha}=\frac{Z_{\alpha}^{1}}{V}
$$

as follows from Eq.(25). For vanishing net strangeness, $S=0$, Eqs.(27)-(29) coincide with those in Ref. [1] (see also references therein).

\section{QUANTUM STATISTICS}

As compared to Eq.(11), in quantum statistics the partition function with exact conservation of net strangeness and with in average conservation of baryonic and electric charges can represented as follows :

$$
Z_{S}=\sum_{\left\{\nu_{\alpha p}\right\}} \delta\left(\sum_{\alpha, p} s_{\alpha} \nu_{\alpha p}-S\right) e^{-\beta \sum_{\alpha, p} \nu_{\alpha p} \varepsilon_{\alpha p}}
$$

where $\varepsilon_{\alpha p}$ is defined by Eq.(2) and occupation numbers take values $\nu_{\alpha p}=0,1, \ldots, K_{\alpha}$ with $K_{\alpha}=1$ for Fermi-Dirac statistics and $K_{\alpha}=\infty$ for Bose-Einstein statistics. By means of Eq.(19) the partition function (31) is reduced to Eq.(20) with partition functions for positive and negative strangeness components given by equation

$$
Z_{S_{\mp}}^{(\mp)}=\sum_{\left\{\nu_{\alpha p}\right\}_{\alpha \in M_{\mp}}} \delta\left(\sum_{\alpha \in M_{\mp}} \sum_{p}\left|s_{\alpha}\right| \nu_{\alpha p}-S_{\mp}\right) e^{-\beta \sum_{\alpha \in M_{\mp}} \sum_{p} \nu_{\alpha p} \varepsilon_{\alpha p}} .
$$

As is shown in Appendix B, the following recurrence relations are fulfilled for these partition functions :

$$
Z_{S_{\mp}}^{(\mp)}=\frac{1}{S_{\mp}} \sum_{\alpha \in M_{\mp}} \sum_{l=1}^{\left[S_{\mp} /\left|s_{\alpha}\right|\right]}\left|s_{\alpha}\right| Z_{\alpha}^{l} Z_{S_{\mp}-l\left|s_{\alpha}\right|}^{(\mp)}=\frac{1}{S_{\mp}} \sum_{s=1}^{s_{\max }} \sum_{l=1}^{\left[S_{\mp} / s\right]} s S_{\mp s, l} Z_{S_{\mp}-l s}^{(\mp)},
$$


where $Z_{0}^{(\mp)}=1$ and new auxiliary functions

$$
\begin{aligned}
S_{\mp s, l} & =\sum_{\alpha \in M_{\mp}} \delta_{s,\left|s_{\alpha}\right|} Z_{\alpha}^{l}, \\
Z_{\alpha}^{l} & =y_{\alpha l} \sum_{p} e^{-\beta l \varepsilon_{\alpha p}} .
\end{aligned}
$$

The quantity $y_{\alpha l}=(-1)^{l+1}$ for Fermi-Dirac statistics and $y_{\alpha l}=1$ for Bose-Einstein statistics. Note that in the case of Maxwell-Boltzmann statistics $y_{\alpha l}=\delta_{l, 1}$. Eq.(33) exactly follows the structure of the corresponding Eq.(22) for classical statistics and can be solved by the recurrence relation method.

The partition function for zero strangeness component looks like (see Appendix B)

$$
Z^{(0)}=\exp \left(\sum_{l=1}^{\infty} S_{0, l}\right)
$$

with $S_{0, l}=\sum_{\alpha \in M_{0}} l^{-1} Z_{\alpha}^{l}$. Notice that under condition $\left|e^{-\beta \varepsilon_{\alpha p}}\right|<1$ the partition function $Z^{(0)}$ can be represented in more familiar form :

$$
Z^{(0)}=\prod_{\alpha \in M_{0}} \prod_{p}\left(1 \pm e^{-\beta \varepsilon_{\alpha p}}\right)^{ \pm 1},
$$

where the upper sign corresponds to Fermi-Dirac statistics and the lower sign does to BoseEinstein one.

As shown in Appendix B, the mean occupation numbers for hadrons with negative and positive strangeness, $\alpha \in M_{\mp}$, are given as

$$
\left\langle\nu_{\alpha p}\right\rangle_{S}=\frac{Z^{(0)}}{Z_{S}} \sum_{S_{+}=0}^{\infty} \sum_{S_{-}=0}^{\infty} \delta\left(-S_{-}+S_{+}-S\right) Z_{S_{ \pm}}^{( \pm)} \sum_{l=1}^{\left[S_{\mp} /\left|s_{\alpha}\right|\right]} y_{\alpha l} e^{-\beta l \varepsilon_{\alpha p}} Z_{S_{\mp}-l\left|s_{\alpha}\right|}^{(\mp)}, \alpha \in M_{\mp},
$$

while those for non-strange particles are

$$
\left\langle\nu_{\alpha p}\right\rangle_{S}=\sum_{l=1}^{\infty} y_{\alpha l} e^{-\beta l \varepsilon_{\alpha p}}, \quad \alpha \in M_{0} .
$$

Under the same condition $\left|e^{-\beta \varepsilon_{\alpha p}}\right|<1$ we arrive at the familiar expression for mean occupation numbers (39) :

$$
\left\langle\nu_{\alpha p}\right\rangle_{S}=\frac{1}{e^{\beta \varepsilon_{\alpha p}} \pm 1}, \quad \alpha \in M_{0} .
$$

To get the average number of particles $\alpha$ one should sum the mean occupation numbers (38) and (39) over momentum $p$. For strange particles $\alpha \in M_{\mp}$ we have

$$
\left\langle n_{\alpha}\right\rangle_{S}=\frac{Z^{(0)}}{Z_{S}} \sum_{S_{+}=0}^{\infty} \sum_{S_{-}=0}^{\infty} \delta\left(-S_{-}+S_{+}-S\right) Z_{S_{ \pm}}^{( \pm)} \sum_{l=1}^{\left[S_{\mp} /\left|s_{\alpha}\right|\right]} Z_{\alpha}^{l} Z_{S_{\mp}-l\left|s_{\alpha}\right|}^{(\mp)}, \quad \alpha \in M_{\mp} .
$$


For non-strange hadrons $\alpha \in M_{0}$ the following relation is valid :

$$
\left\langle n_{\alpha}\right\rangle_{S}=\sum_{l=1}^{\infty} Z_{\alpha}^{l}, \quad \alpha \in M_{0}
$$

After integration the single particle partition function (35) is reduced to (cf. with Eq.(17))

$$
Z_{\alpha}^{l}=y_{\alpha l} \frac{g_{\alpha} V}{2 \pi^{2}} \frac{m_{\alpha}^{2}}{\beta l} K_{2}\left(\beta l m_{\alpha}\right) e^{\beta l\left(\mu_{B} b_{\alpha}+\mu_{Q} q_{\alpha}\right)} .
$$

One should note that all formulae for Maxwell-Boltzmann statistics from the proceeding section are recovered by the simple substitution $y_{\alpha l}=\delta_{l, 1}$ into appropriate quantum relations.

\section{STRANGENESS EXCITATION FUNCTION}

To reveal canonical effects in the obtained statistical relations, let us consider excitation functions for strange particle multiplicities. These multiplicities are calculated along the "experimental" freeze-out curve defined at any bombarding energy by the temperature $T$ and the baryon chemical potential $\mu_{B}$ :

$$
\mu_{B}[\text { in } M e V]=\frac{1270}{1+\sqrt{s} / 4.3}, \quad T[\text { in } M e V]=\frac{170}{1+e^{-0.48(\sqrt{s}-3.8)}}, \quad[\sqrt{s} \text { is in } G e V],
$$

as approximated in [1, 16]. The perfect hadronic gas includes all mesons with $m_{\alpha} \lesssim 1.6 \mathrm{GeV}$ and baryons with $m_{\alpha} \lesssim 2 \mathrm{GeV}$. In calculations of a particular hadron yield the contribution from decays of heavier hadrons is taken into account in addition to the primary yield

$$
\left\langle n_{\alpha}\right\rangle=\left\langle n_{\alpha}\right\rangle^{\text {primary }}+\sum_{\gamma} \operatorname{Br}(\gamma \rightarrow \alpha)\left\langle n_{\gamma}\right\rangle
$$

where the branching ratios $\operatorname{Br}(\gamma \rightarrow \alpha)$ are taken from the Review of Particle Physics [15]. Certainly the final answer will depend on whether only strong decays or also weak ones are considered. The latter circumstance is controlled by the experimental acceptance [16].

The $K^{+} / \pi^{+}$excitation function is shown in Fig.1 for systems formed in high-energy heavy-ion collisions. The curve for the strangeness neutral system described by the classical Maxwell-Boltzmann statistics and without taking into account weak decays reproduces closely the earlier statistical model results (see [6, 7] as well as the review [1]). Note that two curves calculated within the classical ensemble by two above-discussed methods coincide exactly and are indistinguishable in the figure. Calculations have been carried out using 
a complete equation (27). If $s_{\max }=1$ is assumed (i.e., Eq.(29) is applied), which physically implies that associative production of the $K^{+}$mesons is accompanied by creation of single-strange-charge particles only, the $K^{+} / \pi^{+}$ratio decreases by about $5 \%$ at the colliding energy $\sqrt{s} \sim 8 \mathrm{GeV}$.

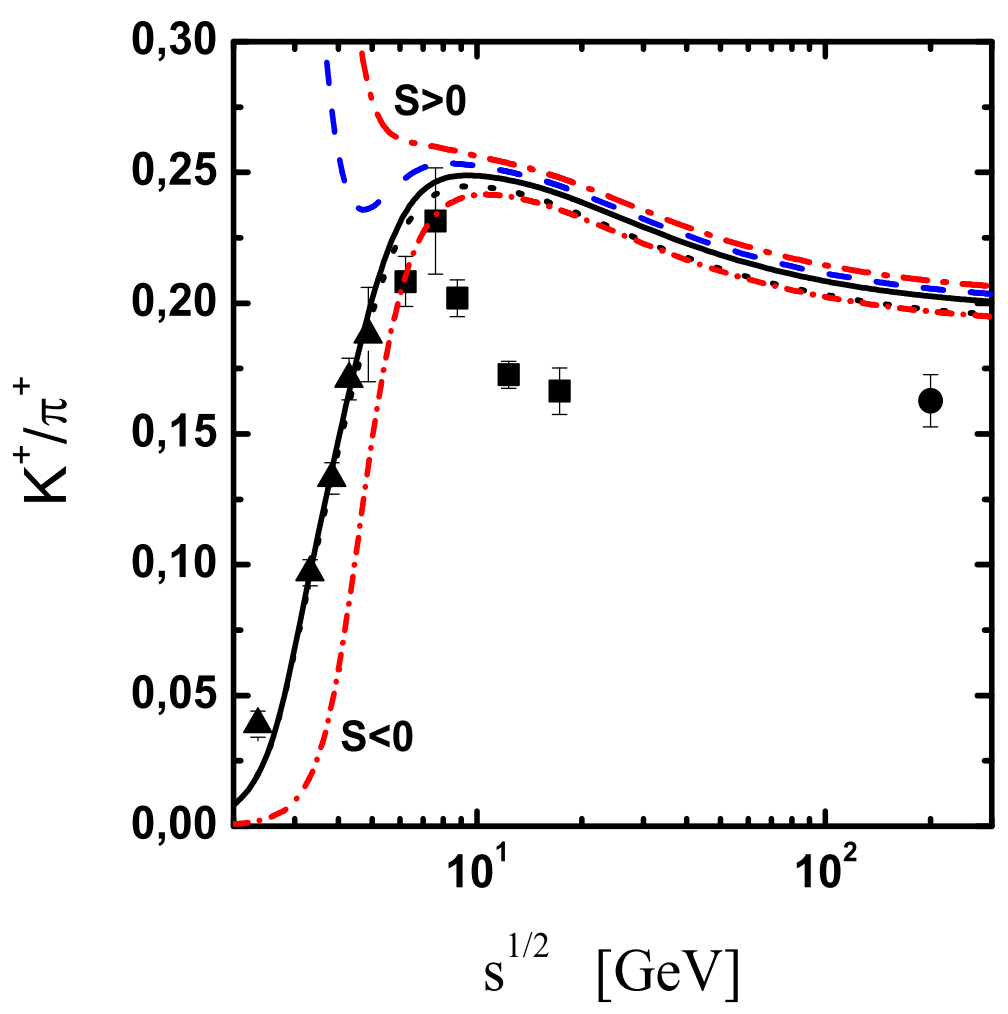

FIG. 1: Excitation function for relative strange particle production, $K^{+} / \pi^{+}$, in heavy ion collisions. The symbols are experimental $4 \pi$ data of E866 (triangles), NA49 (squares) and BRAHMS (circles) collaborations [17, 18, 19]. The lines are thermal model calculations for two types of statistics: classical (solid) and quantum (dotted) for the net strangeness of the system $S=0$ and classical Maxwell-Boltzmann ensemble for systems with non-vanishing net strangeness $S=5$, 10 (long dashed, dash dotted) and $S=-10$ (short dash dotted), respectively. The radius of the strangeness correlated sphere is $R=7 \mathrm{fm}$ and electrical chemical potential $\mu_{Q}=0$ and $s_{\max }=3$. The additional contribution into $K^{+}$and $\pi^{+}$from strong decays of hadrons has been taken into account.

The inclusion of quantum statistics slightly decreases the $K^{+} / \pi^{+}$ratio at $\sqrt{s}>6 \mathrm{GeV}$. As is found, the contribution of weak decays of hadrons with the life-time $\tau<10^{-10}$ sec decreases this ratio by (15)\% without changing the shape of the excitation function in agreement with recent finding in [16]. 
It is noteworthy that due to local strangeness conservation, two volumes are usually introduced. The first $V$ is coming as a normalization factor of the partition function (see, for example, Eq.(30), which is dropped out if the particle ratio is considered. The second volume, $V_{c}$, enters the argument $x$ of the modified Bessel functions $I_{n}(x)$ in Eqs.(27)-(29) and characterizes the range of strangeness correlations. It was shown [1] that for heavy ion collisions $V_{c}$ is proportional to a number of participant nucleons. The presented results correspond to a constant strangeness correlation volume $V_{c}$ treated as a free parameters. Following Ref.[20], this volume is taken as a sphere with the radius of about $R \sim 7 \mathrm{fm}$, which ensures the agreement with experiment for the fast growth of the $K^{+} / \pi^{+}$ratio at moderate energies. Asymptotically, this ratio goes to the GC results being a constant (or slightly depending on a particular choice of the freeze-out state). However, in dynamical models this fall may be energy dependent. In particular, in the expanding fireball model [21] the strangeness correlation volume was associated with that of the initial thermalized state as the Lorentz-contracted cylinder $V_{c}=\pi R^{2} \cdot 2 R / \gamma_{c m}$, where $\gamma_{c m}$ is the $\gamma$-factor of colliding nuclei in the c.m. system. In this case, for high colliding energies the $K^{+} / \pi^{+}$excitation function falls down faster than for a constant volume. Nevertheless, the observed peaked maximum in the excitation function is not reproduced within this dynamical model (see also the comprehensive analysis [18]). It is remarkable that such a behavior has really been predicted in terms of a simple statistical model of the early stage [22] as the onset of the first order deconfinement phase transition. It is of interest to note that the above mentioned calculations, made in a similar scenario but in a more elaborated model of an expanding fireball [21] with various equations of state, do not give rise to a peaked maximum and calculated excitation functions are practically independent of the equation of state. In other words, the condition of global strangeness neutrality at the freeze-out washes out particularities of strangeness evolution at the earlier stage.

Generally speaking, the introduction of two volumes, $V$ and $V_{c}$, into the statistical description based on the empirical freeze-out $T-\mu_{B}$ curve breaks thermodynamical consistency of the model. Indeed, in the presented calculations there is only $V$. On the other hand, the $T$ and $\mu_{B}$ values were extracted from the particle ratios and the volume is not fixed. To correct the description we introduce explicitly the conservation of the total baryon $B$ and 
electric $Q$ charges

$$
\begin{aligned}
& B=\sum_{\alpha}\left\langle n_{\alpha}\right\rangle b_{\alpha}, \\
& Q=\sum_{\alpha}\left\langle n_{\alpha}\right\rangle q_{\alpha} .
\end{aligned}
$$

Since $\mu_{B}$ is known from the $T-\mu_{B}$ curve, the first equation (44) defines the volume of the system and the second one allows one to find the electric charge potential $\mu_{Q}$. The
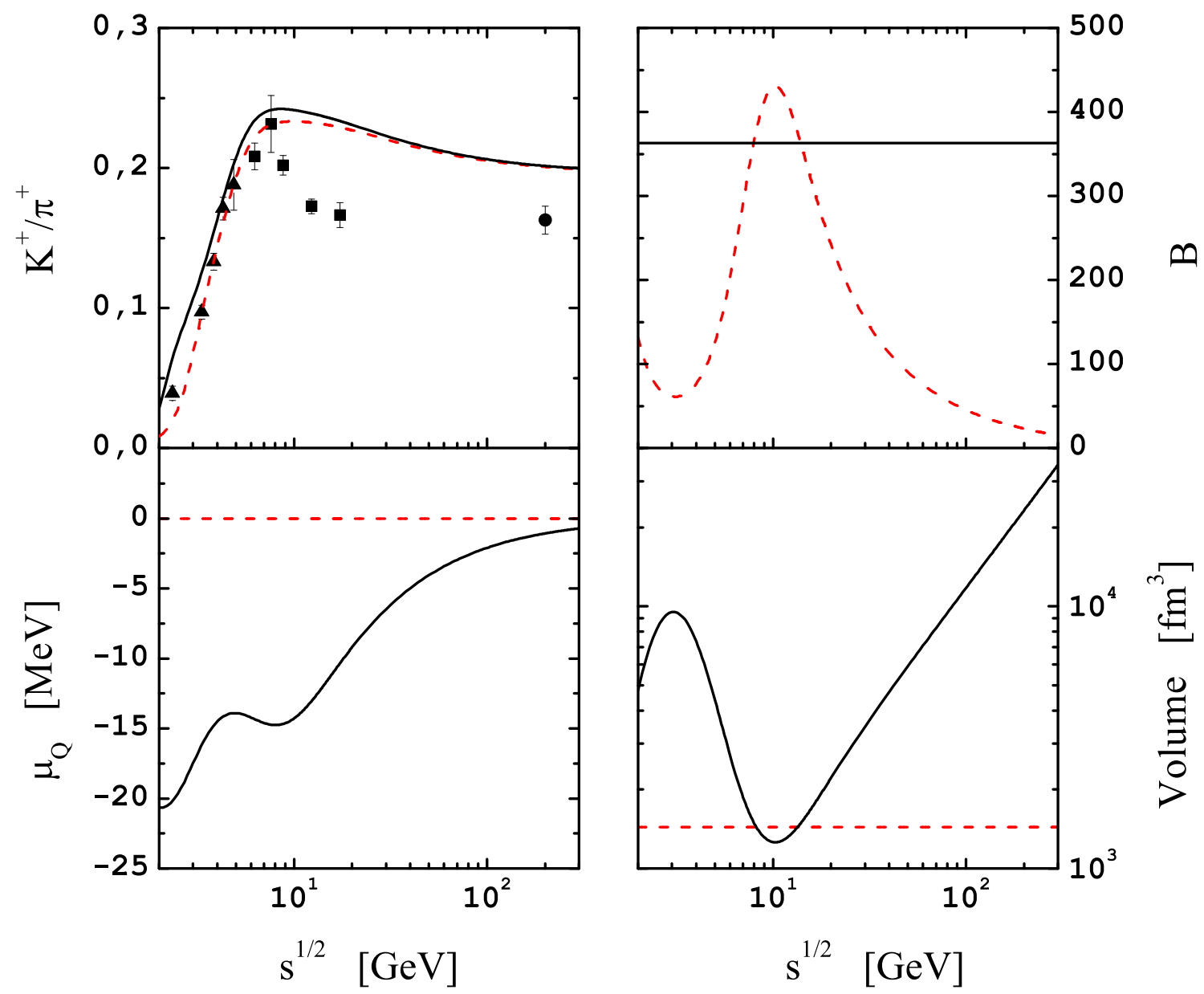

FIG. 2: The $K^{+} / \pi^{+}$excitation function and energy dependence of the total baryon charge $B$, electrical chemical potential $\mu_{Q}$ and volume (sphere radius) for the net strangeness of the system $S=0$. Lines are statistical model calculations for classical statistics and $s_{\max }=1$ (i.e. multistrange hyperons are ignored) at two assumptions : (i) dashed lines $-\mu_{Q}=0$ and correlated sphere radius $R=7 \mathrm{fm}$ (as in Fig.1); (ii) solid lines $-\mu_{Q}$ and $R$ are found from the additional conservation of baryon and electric charges of the system, Eqs.(44) and (45). Experimental points are the same as in Fig.1. 
kaon excitation function recalculated for new obtained volume $V=V_{c}$ is presented in Fig.2 together with some thermodynamic quantities. These results are obtained for $B=364$ and $Q=142$ due to $5 \%$ centrality selection of data.

It is seen that the recalculated $K^{+} / \pi^{+}$ratio does not differ noticeably from that in the case of free correlation volume $V_{c}$, except maybe the SIS energies due to non-vanishing $\mu_{Q}$. The obtained electric chemical potential is not large and it is getting even smaller with increasing the invariant colliding energy $s^{1 / 2}$. However, the baryon conservation is fulfilled only for the energy dependent volume. It is noteworthy that the system volume behaves non-monotonically having a minimum near $s^{1 / 2} \sim 10 \mathrm{GeV}$. This finding may be an evidence of the softest point effect at the passage of the phase boundary. In addition, this behavior roughly correlates with the freeze-out pion volume measured by HBT interference experiments [23].

Coming back to Fig.1, one can compare the $K^{+} / \pi^{+}$excitation function to those calculated within the Maxwell-Boltzmann ensemble but for different values of net strangeness $S$ of the hadronic system. It is not of purely academic interest. As was first noted in [24], in the Gibbs phase coexistence of a baryon-rich quark-gluon plasma there is strangeness separation in phases, the so-called strangeness distillation effect: While the net strangeness of the system is zero, the strangeness of its each component can differ from zero. In particular, in the quark subsystem, being a small admixture to the hadronic one, a number of strange quarks can be larger than that of strange antiquarks (i.e. $S<0$ ) which should be compensated by hadronic phase (i.e. $S>0$ ). A similar situation may occur below the critical temperature for systems with crossover deconfinement phase transition. The distillation effect is even stronger for lower colliding energies, as recently shown in [21]. In contrast to the first order phase transition, here a small quark admixture can survive during the expansion stage until freeze-out. This may give rise to an observable effect if particles from each phase get on shell at freeze-out preserving their strangeness. One should remember that recent lattice QCD calculations with physical quark masses [25] tell us that at $\mu_{B}=360 \pm 40 \mathrm{MeV}$ and $T=162 \pm 2 \mathrm{MeV}$ there is the critical end-point where the crossover changes to a phase transition of the first order. It means that proceeding from high to lower energies (see Fig.1), the hadronic subsystem follows along the curve for $K^{+} / \pi^{+}$with $S>0$ strangeness components, then stops near the end-point and jumps down on the curve with $S=0$. The given parameters of the critical end-point roughly correspond to the bombarding energy 
$\sim 30 \mathrm{GeV}$ [21] and correlate with the position of the maximum for the relative strangeness yield in Fig.1. Therefore, in this scenario the observed maximum is treated as a manifestation of the critical end-point rather than a jump due to the latent heat at the first order phase transition. A change of the nature of phase transition at the critical end-point may manifest itself in other observables as well. Since at this change the dominating hadronic phase of the quark-hadron mixture is enriched with strange antiquarks, effects should be more noticeable in the $K^{+}$component.

\section{CONCLUSIONS}

The derivation of the Maxwell-Boltzmann partition function and ensemble averages with the exact strangeness conservation has been reconsidered in a general case of non-vanishing net strangeness. A new method of recurrence relations was applied to solve the partition function. This method has certain advantages as compared to the numerical method used in [18] for the canonical description of baryon charge conservation of systems with the total baryon charge up to about 100. The recurrence relation technique can be successfully applied to larger systems, as it was earlier demonstrated by a canonical treatment of statistical multifragmentation of excited heavy nuclei [9].

Using the recurrence relations method we first generalize the calculation scheme for the strangeness canonical ensemble to the case of quantum ensemble. Taking into account quantum statistics, the $K^{+} / \pi^{+}$ratio turns out to be slightly lower at collision energies $\sqrt{s} \gtrsim 6 \mathrm{GeV}$.

In the present approach the general behavior of the $K^{+} / \pi^{+}$experimental excitation function is reproduced roughly to the same level of agreement as that in earlier statistical treatments. Uncertainties related to a possible contribution of weak decays and energy dependence of dynamic correlation volume are pointed out. While the initial state of the equilibrium system is taken from the chemical freeze-out $T-\mu_{B}$ curve, an additional account of the baryonic and electric charge conservation does not change noticeably the particle ratio but provides the thermodynamic consistency of the strangeness canonical approach. The obtained unique system volume is energy dependent and exhibits clear minimum near $s^{1 / 2} \sim 10 \mathrm{GeV}$, which deserves further investigations.

Nevertheless, the anomalous increase of this ratio near the bombarding energy of about 30 
$\mathrm{GeV}$, having been interpreted as a sign of deconfinement phase transition, is not reproduced by this model. A new scenario based on the strangeness distillation idea has been proposed where this anomalous strangeness enhancement is considered as a sign of the evolving system passing in vicinity of the critical end-point. Arguments in favor of this hypothesis are given. In this respect, multiplicity fluctuations of rare strange particles can be a measure of chemical equilibrium [26], and therefore are of special interest. A more convincing test needs delicate dynamic calculations which are in progress now.

\section{Acknowledgements:}

We are grateful to B.Friman, Yu.Ivanov and K.Redlich for discussion and valuable remarks. This work was supported in part by the Deutsche Forschungsgemeinschaft (DFG project 436 RUS 113/558/0-2), the Russian Foundation for Basic Research (RFBR grants 03-02-04008) and the Moldavian-U.S. Bilateral Grants Program (CRDF Project MP2-3045).

[1] Braun-Munzinger P, Redlich K and Stachel J in Quark Gluon Plasma 3 eds. Hwa R C and Wang X N, World Scientific, Singapore p.491; nucl-th/0304013

[2] Redlich K and Turko B 1980 Z. Phys. B 97 279; Turko B 1981 Phys. Lett. B 104153

[3] Hagedorn R and Redlich R 1985 Z. Phys. C 27 541; Rafelski J and Danosh D 1980 Phys. Lett. B 97 279; Turko B and Rafelski J 2001Eur. Phys. J. C 18587

[4] Redlich K, Karsch F and Tounsi A 2002 In Physical and Mathematical Aspects of Symmetries Paris p.139; |hep-ph/0302245

[5] Keränen A and Becattini F 2002 J.Phys. G28 2041; nucl-th/0112045

[6] Cleymans J, Redlich R and Suhonen E 1991 Z. Rev. C 51 137; Cleymans J, Oeschler H and Redlich K 2001 Phys. Lett. B 48527

[7] Hamieh S, Redlich K and Taunsi A 2000 Phys. Lett. B 486 61; 2001 J. Phys. G 27413

[8] Reordan J 1958 An Introduction to Combinatorial Analysis Wiley, New York

[9] Parvan A S, Toneev V D and Płoszajczak M 2000 Nucl. Phys. A 676409

[10] Parvan A S 2004 Theor. and Math. Phys. 140977

[11] Keränen A and Becattini F 2002 Phys. Rev. C 65 044901; nucl-th/0112021

[12] Cleymans J Suhonen E and Weber G M 1992 Z. Phys. C 53485

[13] Chase K C and Mekjian A Z 1994 Phys. Rev. C 50 2078; 1995 Phys. Rev. C 52 R2339 
[14] Pratt S and Ruppert J 2003 Phys. Rev. C 68024904

[15] Hagiwara K et al 2002 Phys. Rev. D 66 010001-1

[16] Andronic A and Braun-Munzinger P 2004 hep-ph/0402291

[17] Bratkovskaya E L, Bleicher M, Reiter M, Soff S, Stöcker H, van Leeuwen M, Bass S A and Cassing W 2004 Phys.Rev. C 69054907

[18] Becattini F, Gazdzicki M, Keranen A, Manninen J and Stock R 2004 Phys.Rev. C 69 024905; hep-ph/0310049

[19] Botje M, for the NA49 Collaboration 2004 nucl-ex/0407004

[20] Taunsi A and Redlich K 2002 J. Phys. G 282095

[21] Toneev V D, Nikonov E G, Friman B, Nörenberg W and Redlich K 2004 Eur. Phys. J. C32 399; hep-ph/0308088

[22] Gazdzicky M and Gorenstein M I 1999 Acta Phys. Pol. B 30 2705; hep-ph/9803462

[23] Tomasik B and Wiedemann U A in Quark Gluon Plasma 3, eds. Hwa R C and Wang X N, World Scientific, Singapore; nucl-th/0304013

[24] Subramanian P R, Stöcker H and Greiner W 1986 Phys. Lett. 173 468; Greiner C, Koch P and Stöcker H 1987 Phys. Rev. Lett. 58 1825; 1988 Nucl. Phys. A 479 295c

[25] Fodor Z and Katz S D 2004 JHEP 404 50; hep-lat/0402006

[26] Jeon S, Koch V, Redlich K and Wang X N 2002 Nucl.Phys. A697 546; nucl-th/0105035

\section{APPENDIX A: RECURSIVE RELATIONS IN CLASSICAL STATISTICS}

Let us prove the recurrence relations (22) for the classical perfect gas partition function with positive and negative components of the net strangeness. It is easy to see that for the given species $\alpha$ in a quantum state $p$ the sum over occupation numbers $\nu_{\alpha p}$ satisfies the following identity [10]

$$
\begin{aligned}
& \sum_{\nu_{\alpha p}=0}^{\infty} \delta\left(\sum_{\alpha^{\prime}, p^{\prime}} s_{\alpha^{\prime}} \nu_{\alpha^{\prime} p^{\prime}}-S\right) \nu_{\alpha p} \frac{1}{\nu_{\alpha p} !} e^{-\beta \nu_{\alpha p} \varepsilon_{\alpha p}}= \\
& \quad=e^{-\beta \varepsilon_{\alpha p}} \sum_{\nu_{\alpha p}=0}^{\infty} \delta\left(\sum_{\alpha^{\prime}, p^{\prime}} s_{\alpha^{\prime}} \nu_{\alpha^{\prime} p^{\prime}}-\left(S-s_{\alpha}\right)\right) \frac{1}{\nu_{\alpha p} !} e^{-\beta \nu_{\alpha p} \varepsilon_{\alpha p}} .
\end{aligned}
$$

Taking into account the Kronecker symbol, Eq.(21) can be rewritten as

$$
Z_{S_{\mp}}^{(\mp)}=\frac{1}{S_{\mp}} \sum_{\alpha^{\prime} \in M_{\mp}} \sum_{p^{\prime}}\left|s_{\alpha^{\prime}}\right| \sum_{\left\{\nu_{\alpha p}\right\}_{\alpha \in M_{\mp}}} \delta\left(\sum_{\alpha \in M_{\mp}} \sum_{p}\left|s_{\alpha}\right| \nu_{\alpha p}-S_{\mp}\right) \times
$$




$$
\times \nu_{\alpha^{\prime} p^{\prime}} \frac{1}{\prod_{\alpha \in M_{\mp}} \prod_{p} \nu_{\alpha p} !} e^{-\beta \sum_{\alpha \in M_{\mp}} \sum_{p} \nu_{\alpha p} \varepsilon_{\alpha p}}
$$

Using identity (A1), the summation over $\nu_{\alpha p}$ gives

$$
\begin{gathered}
Z_{S_{\mp}}^{(\mp)}=\frac{1}{S_{\mp}} \sum_{\alpha^{\prime} \in M_{\mp}} \sum_{p^{\prime}}\left|s_{\alpha^{\prime}}\right| e^{-\beta \varepsilon_{\alpha^{\prime} p^{\prime}}} \sum_{\left\{\nu_{\alpha p}\right\}_{\alpha \in M_{\mp}}} \delta\left(\sum_{\alpha \in M_{\mp}} \sum_{p}\left|s_{\alpha}\right| \nu_{\alpha p}-\left(S_{\mp}-\left|s_{\alpha^{\prime}}\right|\right)\right) \times \\
\times \frac{1}{\prod_{\alpha \in M_{\mp}} \prod_{p} \nu_{\alpha p} !} e^{-\beta \sum_{\alpha \in M_{\mp}} \sum_{p} \nu_{\alpha p} \varepsilon_{\alpha p}} .
\end{gathered}
$$

By means of Eq.(1) the recurrence relations (A3) can be obtained directly [9, 10]

$$
Z_{S_{\mp}}^{(\mp)}=\frac{1}{S_{\mp}} \sum_{\alpha \in M_{\mp}} \sum_{p}\left|s_{\alpha}\right| e^{-\beta \varepsilon_{\alpha p}} Z_{S_{\mp}-\left|s_{\alpha}\right|}^{(\mp)} .
$$

Thus, the formulae (22) has been proved.

Mean occupation numbers are evidently given as

$$
\left\langle\nu_{\alpha^{\prime} p^{\prime}}\right\rangle_{S}=\frac{1}{Z_{S}} \sum_{\left\{\nu_{\alpha p}\right\}} \delta\left(\sum_{\alpha, p} s_{\alpha} \nu_{\alpha p}-S\right) \nu_{\alpha^{\prime} p^{\prime}} \frac{1}{\prod_{\alpha, p} \nu_{\alpha p} !} e^{-\beta \sum_{\alpha, p} \nu_{\alpha p} \varepsilon_{\alpha p}}
$$

To sum here over $\nu_{\alpha^{\prime} p^{\prime}}$ the identity (A1) should be used :

$$
\begin{aligned}
\left\langle\nu_{\alpha^{\prime} p^{\prime}}\right\rangle_{S}=e^{-\beta \varepsilon_{\alpha^{\prime} p^{\prime}}} & \frac{1}{Z_{S}} \sum_{\left\{\nu_{\alpha p}\right\}} \delta\left(\sum_{\alpha, p} s_{\alpha} \nu_{\alpha p}-\left(S-s_{\alpha^{\prime}}\right)\right) \frac{1}{\prod_{\alpha, p} \nu_{\alpha p} !} e^{-\beta \sum_{\alpha, p} \nu_{\alpha p} \varepsilon_{\alpha p}}= \\
= & e^{-\beta \varepsilon_{\alpha^{\prime} p^{\prime}}} \frac{Z_{S-s_{\alpha^{\prime}}}}{Z_{S}}
\end{aligned}
$$

where Eq.(11) has been used for getting the latter equality.

\section{APPENDIX B: RECURSIVE RELATIONS IN QUANTUM STATISTICS}

Here we will prove the recurrence relations (33) for the quantum partition functions with positive and negative components of net strangeness. It is readily seen that the following identity for occupation number summing is valid [10]

$$
\begin{aligned}
& \sum_{\nu_{\alpha p}=0}^{K_{\alpha}} \nu_{\alpha p} e^{-\beta \nu_{\alpha p} \varepsilon_{\alpha p}} \delta\left(\sum_{\alpha^{\prime}, p^{\prime}}\left|s_{\alpha^{\prime}}\right| \nu_{\alpha^{\prime} p^{\prime}}-S_{ \pm}\right)= \\
& \quad=\sum_{l=1}^{\left[S_{ \pm} /\left|s_{\alpha}\right|\right]} y_{\alpha l} e^{-\beta l \varepsilon_{\alpha p}} \sum_{\nu_{\alpha p}=0}^{\min \left(\left[S_{ \pm} /\left|s_{\alpha}\right|\right]-l, K_{\alpha}\right)} e^{-\beta \nu_{\alpha p} \varepsilon_{\alpha p}} \delta\left(\sum_{\alpha^{\prime}, p^{\prime}}\left|s_{\alpha^{\prime}}\right| \nu_{\alpha^{\prime} p^{\prime}}-\left(S_{ \pm}-l\left|s_{\alpha}\right|\right)\right)
\end{aligned}
$$


Using the Kronecker symbol, we can rewrite Eq.(32) :

$$
Z_{S_{\mp}}^{(\mp)}=\frac{1}{S_{\mp}} \sum_{\alpha^{\prime} \in M_{\mp}} \sum_{p^{\prime}}\left|s_{\alpha^{\prime}}\right| \sum_{\left\{\nu_{\alpha p}\right\}_{\alpha \in M_{\mp}}} \delta\left(\sum_{\alpha \in M_{\mp}} \sum_{p}\left|s_{\alpha}\right| \nu_{\alpha p}-S_{\mp}\right) \nu_{\alpha^{\prime} p^{\prime}} e^{-\beta \sum_{\alpha \in M_{\mp}} \sum_{p} \nu_{\alpha p} \varepsilon_{\alpha \alpha}}(
$$

Applying identity (B1) for summing over $\nu_{\alpha p}$, we have

$$
\begin{aligned}
& Z_{S_{\mp}}^{(\mp)}=\frac{1}{S_{\mp}} \sum_{\alpha^{\prime} \in M_{\mp}} \sum_{p^{\prime}}\left|s_{\alpha^{\prime}}\right| \sum_{l=1}^{\left[S_{\mp} /\left|s_{\alpha^{\prime}}\right|\right]} y_{\alpha^{\prime} l} e^{-\beta l \varepsilon_{\alpha^{\prime} p^{\prime}}} \\
& \times \sum_{\left\{\nu_{\alpha p}\right\}_{\alpha \in M_{\mp}}} \delta\left(\sum_{\alpha \in M_{\mp}} \sum_{p}\left|s_{\alpha}\right| \nu_{\alpha p}-\left(S_{\mp}-l\left|s_{\alpha^{\prime}}\right|\right)\right) e^{-\beta \sum_{\alpha \in M_{\mp}} \sum_{p} \nu_{\alpha p} \varepsilon_{\alpha p}} .
\end{aligned}
$$

The use of (31) in Eq.(B3) immediately gets the recurrence relations [9, 10]

$$
Z_{S_{\mp}}^{(\mp)}=\frac{1}{S_{\mp}} \sum_{\alpha \in M_{\mp}} \sum_{p}\left|s_{\alpha}\right| \sum_{l=1}^{\left[S_{\mp} /\left|s_{\alpha}\right|\right]} y_{\alpha l} e^{-\beta l \varepsilon_{\alpha p}} Z_{S_{\mp}-l\left|s_{\alpha}\right|}^{(\mp)},
$$

which completes the derivation of Eq.(33).

The partition function with zero strangeness component has the form

$$
Z^{(0)}=\sum_{\left\{\nu_{\alpha p}\right\}_{\alpha \in M_{0}}} e^{-\beta \sum_{\alpha \in M_{0}} \sum_{p} \nu_{\alpha p} \varepsilon_{\alpha p}} .
$$

This partition function (B5) can be represented as the product of sums for every species $\alpha$ and then, accounting for the Kronecker symbols, be reduced to the equation

$$
Z^{(0)}=\prod_{\alpha \in M_{0}} \sum_{n_{\alpha}=0}^{\infty} \sum_{\left\{\nu_{\alpha p}\right\}^{\prime}} \delta\left(\sum_{p} \nu_{\alpha p}-n_{\alpha}\right) e^{-\beta \sum_{p} \nu_{\alpha p} \varepsilon_{\alpha p}}
$$

where $\left\{\nu_{\alpha p}\right\}^{\prime}$ is the set of occupation numbers for a fixed species $\alpha$. Formally, sums over occupation numbers $\left\{\nu_{\alpha p}\right\}^{\prime}$ in Eq.(B⿺辶) are quantum single-particle partition function $n_{\alpha}$ of identical particles in the canonical ensemble (see [9, 10]). So, Eq.(B6) can be represented as follows

$$
Z^{(0)}=\prod_{\alpha \in M_{0}} \sum_{n_{\alpha}=0}^{\infty} \sum_{\left\{N_{\alpha l}\right\}^{\prime}} \delta\left(\sum_{l=1}^{n_{\alpha}} l N_{\alpha l}-n_{\alpha}\right) \prod_{l=1}^{n_{\alpha}} \frac{\left(Z_{\alpha}^{l}\right)^{N_{\alpha l}}}{l^{N_{\alpha l}} N_{\alpha l} !} .
$$

After simple transformations we finally get

$$
Z^{(0)}=\prod_{\alpha \in M_{0}} \prod_{l=1}^{\infty} e^{l^{-1}} Z_{\alpha}^{l} .
$$

Mean occupation number are given by the following expression

$$
\left\langle\nu_{\alpha p}\right\rangle_{S}=\frac{1}{Z_{S}} \sum_{\left\{\nu_{\alpha p}\right\}} \delta\left(\sum_{\alpha, p} s_{\alpha} \nu_{\alpha p}-S\right) \nu_{\alpha p} e^{-\beta \sum_{\alpha, p} \nu_{\alpha p} \varepsilon_{\alpha p}}=-\frac{1}{\beta} \frac{\partial \ln Z_{S}}{\partial \varepsilon_{\alpha p}} .
$$


With (19), from here we get for mean occupation numbers with $\alpha \in M_{\mp}$

$$
\begin{aligned}
\left\langle\nu_{\alpha p}\right\rangle_{S} & =\frac{Z^{(0)}}{Z_{S}} \sum_{S_{+}=0}^{\infty} \sum_{S_{-}=0}^{\infty} \delta\left(-S_{-}+S_{+}-S\right) Z_{S_{ \pm}}^{( \pm)} \times \\
& \times \sum_{\left\{\nu_{\alpha p}\right\}_{\alpha \in M_{\mp}}} \delta\left(\sum_{\alpha \in M_{\mp}} \sum_{p}\left|s_{\alpha}\right| \nu_{\alpha p}-S_{\mp}\right) \nu_{\alpha p} e^{-\beta \sum_{\alpha \in M_{\mp}} \sum_{p} \nu_{\alpha p} \varepsilon_{\alpha p}}, \quad \alpha \in M_{\mp}(\mathrm{B} 10)
\end{aligned}
$$

Using identity (B1) and following the derivation of Eqs.(B2) - (B4) from Eq.(B10), we arrive at Eq.(38) for the mean occupation numbers.

In the case of $\alpha \in M_{0}$, the mean occupation numbers are given by the equation

$$
\left\langle\nu_{\alpha p}\right\rangle_{S}=\frac{1}{Z^{(0)}} \sum_{\left\{\nu_{\alpha p}\right\}_{\alpha \in M_{0}}} \nu_{\alpha p} e^{-\beta \sum_{\alpha \in M_{0}} \sum_{p} \nu_{\alpha p} \varepsilon_{\alpha p}}=-\frac{1}{\beta} \frac{\partial \ln Z^{(0)}}{\partial \varepsilon_{\alpha p}}, \quad \alpha \in M_{0} .
$$

If the partition function (B8) for the zero strangeness component is substituted into differential relation (B11), then we arrive at Eq.(39) for mean occupation numbers with $\alpha \in M_{0}$. 